\title{
NUMERICAL EVALUATION OF CAUCHY PRINCIPAL VALUE INTEGRALS WITH SINGULAR INTEGRANDS
}

\author{
PHILIP RABINOWITZ
}

\begin{abstract}
Convergence results are proved for sequences of interpolatory integration rules for Cauchy principal value integrals of the form

$$
f_{-1}^{1} k(x)(f(x) /(x-\lambda)) d x, \quad-1<\lambda<1
$$

when $f(x)$ is singular at a point $\xi \neq \lambda$ and the singularity is ignored or avoided.
\end{abstract}

\section{INTRODUCTION}

In this paper we shall be concerned with the numerical evaluation of Cauchy principal value (CPV) integrals of the form

$$
I(k f ; \lambda):=f_{-1}^{1} k(x) \frac{f(x)}{x-\lambda} d x, \quad-1<\lambda<1,
$$

where $k$ and $f$ are such that $I(k f ; \lambda)$ exists but where $f$ is unbounded in the neighborhood of a point $\xi \in J:=[-1,1]$ with $\xi \neq \lambda$. The purpose of this paper is to determine those cases in which the numerical method is decoupled with respect to the two singular points $\xi$ and $\lambda$, and those in which there is an influence of one singularity on the other.

A particular choice of $k$ occurs when $k=v$, where $v \in A$, the set of all admissible weight functions, i.e., $v \geq 0$ on $J$ and $0<\|v\|_{1}<\infty$. A special $v \in A$ for which we will give most of our specific results is the generalized smooth Jacobi weight function, $v \in \mathrm{GSJ}$, defined by

$$
v(x)=\psi(x) \prod_{j=0}^{p+1}\left|x-t_{j}\right|^{\gamma_{j}}, \quad \gamma_{j}>-1, j=0, \ldots, p+1,
$$

where $-1=t_{0}<t_{1}<\cdots<t_{p+1}=1, p \geq 0$ and $\psi>0, \psi \in D T(J)$. The set of functions $D T(I)$ for any finite interval $I$ of length $l(I)$ is defined by

$$
D T(I):=\left\{g: \int_{0}^{l(I)} \omega_{I}(g ; t) t^{-1} d t<\infty\right\},
$$

Received February 22, 1989.

1980 Mathematics Subject Classification (1985 Revision). Primary 65D30, 65D32.

Key words and phrases. Cauchy principal value integrals, interpolatory integration rules, product integration rules, singular integrands, generalized smooth Jacobi weight. 
where the modulus of continuity $\omega_{I}(g ; t)$ is given by

$$
\omega_{I}(g ; t):=\sup _{\substack{\left|x_{1}-x_{2}\right| \leq t \\ x_{1} \cdot x_{2} \in I}}\left|g\left(x_{1}\right)-g\left(x_{2}\right)\right| .
$$

The numerical integration rules investigated are principally the interpolatory rules based on sets of points $X_{n}:=\left\{x_{i n}: i=1, \ldots, n ; x_{i n} \neq x_{j n}\right.$ if $\left.i \neq j\right\}$, possibly augmented by the point $\lambda$. In many cases $X_{n}$ will be the set of Gauss, Radau or Lobatto points with respect to some $w \in A$, i.e., the zeros of

$$
(1-x)^{r}(1+x)^{s} p_{n-r-s}\left(x ;(1-x)^{r}(1+x)^{s} w\right), \quad r, s \in\{0,1\},
$$

where $\left\{p_{m}(x ; v)=A_{m} x^{m}+\cdots ; A_{m}>0\right\}$ is the sequence of orthonormal polynomials with respect to $v$. When $k=v$, it is usual to choose $w=v$.

Interpolatory rules for CPV integrals have been studied extensively by many authors, both from theoretical and practical points of view $[1,3,6,9]$. In $\S 2$ we shall give the main convergence results for these rules. We shall also give results on the numerical integration of ordinary integrals where the integrand is an improperly integrable function belonging to the class $M_{d}(\xi ; k)$ where $-1 \leq \xi<1$. As in [10],

$$
M_{d}(\xi ; k):=\{f: f \in C(\xi, 1],|f(x)| \leq F(x) \text { in } J, F=0 \text { in }[-1, \xi] \text {, }
$$

$F$ is nonnegative, continuous and nondecreasing

$$
\text { in } \left.(\xi, 1] \text { and } k F \in L_{1}(J)\right\} \text {. }
$$

For such functions $f \in M_{d}(\xi ; k)$, various results are known about the convergence to the (improper) integral of $f$ of numerical integration rules which either ignore or avoid the singularity at $\xi$.

In $\S 3$ we shall study the convergence of interpolatory rules for $I(k f ; \lambda)$ based on $X_{n}$ when $f \in M_{d}(\xi ; k)$. These rules have a very favorable convergence behavior for continuous functions for certain choices of $X_{n}$ and $k$, in particular when $k=w \in$ GSJ . Surprisingly, in the singular situation there are problems when $w \in$ GSJ and the $x_{\text {in }}$ are zeros of (3) in the case when $\xi=-1$, and only then. This is in contrast to the usual experience in numerical integration in the presence of a singularity where there is much better convergence behavior when $\xi$ is an endpoint than when $\xi$ is an interior point; see e.g. [10].

In $\S 4$ we shall investigate the convergence of more general integration rules for $I(k f ; \lambda)$ when $f \in M_{d}(\xi ; k)$, a special case of which are the interpolatory rules based on the set $X_{n} \cup\{\lambda\}$ with $\lambda \neq x_{i n}, i=1, \ldots, n$. For $f \in C$, the convergence behavior of these interpolatory rules is much more problematic than in the case of rules based only on $X_{n}$. However, the complications imposed by the singularity in $f$ do not introduce additional difficulties. In fact, both in the case discussed in $\S 3$ with $\xi \neq-1$ and in the case discussed in $\S 4$, the influences of the singularities at $\xi$ and at $\lambda$ are decoupled. Only when $\xi=-1$ is there an interaction between the two singularities.

\section{Preliminaries}

Let $f \in M_{d}(\xi ; k)$ and let $\lambda \in(\xi, 1)$, since otherwise $I(k f ; \lambda)$ is not a CPV integral. We define $N_{\delta}(\lambda):=[\lambda-\delta, \lambda+\delta]$, where we restrict $\delta$ so that 
$N_{\delta}(\lambda) \subset(\xi, 1)$. It then follows by an argument similar to one involving $f \in C(J)$ that if $k, f \in D T\left(N_{\delta}(\lambda)\right)$, then $I(k f ; \lambda)$ exists (see $\left.\S 3\right)$. Assume now that we are given an arbitrary set $X_{n}$ and a set of weights $w_{i n}(k)$, $i=1, \ldots, n$, in the interpolatory product integration rule (IPIR)

$$
I(k g):=\int_{-1}^{1} k(x) g(x) d x=I_{n}(k g)+E_{n}(g),
$$

where

$$
I_{n}(k g):=\sum_{i=1}^{n} w_{i n}(k) g\left(x_{i n}\right)
$$

and where $E_{n}(g)=0$ whenever $g \in P_{n-1}$, the set of polynomials of degree $\leq n-1$. If we define $w_{i n}(k ; \lambda)$ by

$$
w_{i n}(k ; \lambda):=\frac{w_{i n}(k)-I\left(k P_{n} ; \lambda\right) / P_{n}^{\prime}\left(x_{i n}\right)}{x_{i n}-\lambda},
$$

where

$$
P_{n}(x):=\prod_{i=1}^{n}\left(x-x_{i n}\right)
$$

and define

$$
I_{n}(k g ; \lambda):=\sum_{i=1}^{n} w_{i n}(k ; \lambda) g\left(x_{i n}\right)
$$

then

$$
I(k g ; \lambda)=I_{n}(k g ; \lambda)+E_{n}(g ; \lambda)
$$

and $E_{n}(g ; \lambda)=0$ whenever $g \in P_{n-1}$. The $w_{i n}(k ; \lambda)$ can be computed in a stable manner once $I(k ; \lambda)$ is known [6].

We have the following estimates for $E_{n}(g ; \lambda)$ in terms of $\tilde{E}_{n}(g)$ defined by $\tilde{E}_{n}(g):=\left\|g-t_{n}(g)\right\|$, where $t_{n}(g ; x) \in P_{n}$ is the polynomial of best approximation to $g$.

If $k \in D T\left(N_{\delta}(\lambda)\right) \cap L_{1}(J)$ and $g \in D T\left(N_{\delta}(\lambda)\right) \cap C(J)$, then

$$
E_{n}(g ; \lambda)=O\left(\Lambda\left(X_{n}\right) \log n \tilde{E}_{n-1}(g)\right),
$$

where $\Lambda\left(X_{n}\right)$ is the Lebesgue constant of $X_{n}$ with respect to Lagrange interpolation (cf. $[1,(2.12)]$ ), and furthermore

$$
E_{n}(g ; \lambda)=O\left(\sum_{i=1}^{n}\left|w_{i n}(k ; \lambda)\right|+\log n\right) \tilde{E}_{n-1}(g) .
$$

If $k=w \in \mathrm{GSJ}, x_{i n}$ are the zeros of (3) and $\lambda \neq t_{j}, j=1, \ldots, p$, as we shall always assume hereafter, then $\sum_{i=1}^{n}\left|w_{i n}(k ; \lambda)\right|=O(\log n)$ [1], so that

$$
E_{n}(g ; \lambda)=O\left(\log n \tilde{E}_{n-1}(g)\right) \text {. }
$$


If $k \in D T\left(N_{\delta}(\lambda)\right) \cap L_{1}(J)$, where $\delta$ is such that $t_{j} \notin N_{\delta}(\lambda), j=1, \ldots, p$, and satisfies

$$
k \log |k| \in L_{1}(J), \quad k w^{-1 / 2}\left(1-x^{2}\right)^{-1 / 4} \in L_{1}(J)
$$

for some $w \in \mathrm{GSJ}$, and if $x_{i n}$ are the zeros of (3), then (13) holds [9]. In what follows, we shall always assume that $k, X_{n}$ and $f$ are such that $E_{n}(f ; \lambda)=$ $o(1)$.

If we replace $f$ by the polynomial interpolating $f$ at the points $X_{n} \cup\{\lambda\}$ with $\lambda \neq x_{i n}, i=1, \ldots, n$, then we get the approximation

$$
I(k f ; \lambda)=\widehat{I}_{n+1}(k f ; \lambda)+\widehat{E}_{n+1}(f ; \lambda),
$$

where

$$
\widehat{I}_{n+1}(k f ; \lambda):=\sum_{i=1}^{n} w_{i n}(k) \frac{f\left(x_{i n}\right)}{x_{i n}-\lambda}+\frac{I\left(k P_{n} ; \lambda\right)}{P_{n}(\lambda)}
$$

and $\widehat{E}_{n+1}(f ; \lambda)=0$ if $f \in P_{n}$, except that if $k=w \in A$ and $x_{i n}$ are the zeros of (3), then $\widehat{E}_{n+1}(f ; \lambda)=0$ if $f \in P_{2 n-r-s}$. Since it can be shown that

$$
\hat{I}_{n+1}(k f ; \lambda)=\sum_{i=1}^{n} w_{i n}(k) f_{\lambda}\left(x_{i n}\right)+f(\lambda) I(k ; \lambda)
$$

and

$$
I(k f ; \lambda)=I\left(k f_{\lambda}\right)+f(\lambda) I(k ; \lambda),
$$

where

$$
f_{\lambda}(x):= \begin{cases}(f(x)-f(\lambda)) /(x-\lambda), & x \neq \lambda, \\ f^{\prime}(\lambda), & x=\lambda, f^{\prime}(\lambda) \text { exists, } \\ 0, & \text { otherwise, }\end{cases}
$$

it follows that $\widehat{E}_{n+1}(f ; \lambda)=o(1)$ if the IPIR $I_{n}\left(k f_{\hat{\lambda}}\right)$ converges to $I\left(k f_{\lambda}\right)$.

We see from (17) and (18) that one can use any product integration rule (PIR) to approximate (18) and not necessarily an IPIR, i.e., the weights $w_{i n}(k)$ in (17) may be replaced by the weights $\bar{w}_{i n}(k)$ in the arbitrary PIR

$$
\bar{I}_{n}(k g):=\sum_{i=1}^{n} \bar{w}_{i n}(k) g\left(x_{i n}\right)
$$

and the convergence in the CPV case for $f$ will follow from the convergence of $(20)$ for $f_{\lambda}$. For future reference, we define the companion rule to (6) by

$$
\left|I_{n}\right|(k g):=\sum_{i=1}^{n}\left|w_{\text {in }}(k)\right| g\left(x_{\text {in }}\right) .
$$

The convergence of IPIR's for (bounded) Riemann-integrable functions has been studied by several authors, and their results are summarized in the following two theorems [11]. 
Theorem A. Let $w \in A$ and $x_{i n}$ be the zeros of (3). Then

$$
I_{n}(k g) \rightarrow I(k g) \quad \text { and } \quad\left|I_{n}\right|(k g) \rightarrow I(|k| g) \quad \text { as } n \rightarrow \infty
$$

for all $g \in R[J]$, the set of all Riemann-integrable functions on $J$, if $k^{2} w^{-1} \in$ $L_{1}(J)$.

Theorem B. Let $w \in G S J$ and $x_{i n}$ be the zeros of

$$
(1-x)^{r}(1+x)^{s} p_{n-r-s}(x ; w), \quad r, s \in\{0,1\} ;
$$

then (22) holds for all $g \in R[J]$ if, for some $p>1$,

(a) $k \in L_{p}$,

(b) $(1-x)^{-r+1 / 4}(1+x)^{-s+1 / 4} w^{1 / 2} \in L_{1}$,

(c) $k(1-x)^{r-1 / 4}(1+x)^{s-1 / 4} w^{-1 / 2} \in L_{p}$.

In [12], there is a result analogous to (11) for IPIR's, namely, that if $g \in C(J)$ and $k \in L_{1}(J)$, then

$$
E_{n}(k g)=O\left(\Lambda\left(X_{n}\right) \tilde{E}_{n-1}(g)\right) .
$$

For the convergence of generalized piecewise polynomial PIR's defined in [10] we have that $I_{n}(k g) \rightarrow I(k g)$ as $n \rightarrow \infty$ for all $g \in P C[J]$, the set of piecewise continuous functions in $J$.

We now give some results on ignoring and avoiding the singularity in integrating functions in $M_{d}(\xi ; k)$. Our first result is basic to future developments.

Theorem C [10]. Let $\xi \in[-1,1)$ and $f \in M_{d}(\xi ; k)$. Assume that $\bar{I}_{n}(k g) \rightarrow$ $I(k g)$ for all $g \in P C[J]$. Then a necessary and sufficient condition that $\bar{I}_{n}(k f) \rightarrow I(k f)$ is that, given $\varepsilon>0$ and $\eta \in(\xi, 1)$, there exists $\beta \in(\xi, \eta)$ such that for all $n$ sufficiently large

$$
\left|\sum_{x_{i n} \leq \beta} \bar{w}_{i n}(k) f\left(x_{i n}\right)\right|<\varepsilon .
$$

The corollaries in [10] are also of interest. In the following two corollaries, we assume that $-1:=x_{0 n}<x_{1 n}$. Similar results exist for the case $x_{1 n}=-1$.

Corollary C.1. Assume that $\bar{I}_{n}(k g) \rightarrow I(k g)$ for all $g \in P C[J]$ and suppose there exists a positive integer $N$, a point $B \in(-1,1]$ and a positive constant $c$ such that

$$
\left|\bar{w}_{i n}(k)\right| \leq c \int_{x_{t-1, n}}^{x_{t n}}|k(x)| d x
$$

for all $n \geq N$ and all $i$ such that $-1<x_{\text {in }} \leq B$. Then $\bar{I}_{n}(k f) \rightarrow I(k f)$ for all $f \in M_{d}(-1 ; k)$.

Corollary C.2. Let $\xi \in(-1,1)$ and assume that $\bar{I}_{n}(k g) \rightarrow I(k g)$ for all $g \in$ $P C[J]$. Suppose there exists a point $B \in\left(\xi_{0}, 1\right]$ and a positive constant $c$ such 
that (25) holds for all $n$ sufficiently large and all $i$ such that $x_{\kappa n}<x_{i n} \leq B$. Then $\bar{I}_{n}^{\prime}(k f) \rightarrow I(k f)$ for all $f \in M_{d}(\xi ; k)$. Consequently, $\bar{I}_{n}(k f) \rightarrow I(k f)$ if and only if $w_{\kappa n} f\left(x_{\kappa n}\right)=o(1)$.

Here, the index $\kappa$ is such that $x_{\kappa-1, n} \leq \xi<x_{\kappa n}, \xi_{0}=\lim \sup _{n \rightarrow \infty} x_{\kappa n}$ and $\bar{I}_{n}^{\prime}(k f):=\bar{I}_{n}(k f)-\bar{w}_{\kappa n} f\left(x_{\kappa n}\right)$, so that the approximation to $I(k f)$ is given by $\sum_{i=\kappa+1}^{n} \bar{w}_{i n} f\left(x_{i n}\right)$, a case of avoiding the singularity, in contrast to Corollary C.1 where we ignore the singularity.

A consequence of these corollaries is that if $w \in$ GSJ and we consider the Gauss rule $G_{n} f$ for $I(w f)$, then $G_{n} f \rightarrow I(w f)$ for all $f \in M_{d}(1 ; w)$ $G_{n}^{\prime} f \rightarrow I(w f)$ for all $f \in M_{d}(\xi ; w)$. This results from the properties of the Gauss points and weights with respect to $w$ as given, say, in [5]. Furthermore, if $w$ is the Jacobi weight function

$$
w(x):=(1-x)^{\alpha}(1+x)^{\beta}, \quad \alpha, \beta>-1,
$$

then, if $f \in M_{d}(\xi ; w)$ and $\xi=\cos \pi p / q, p, q$ integers, then $G_{n} f \rightarrow I(w f)$, while if $\xi=\cos \pi \tau, \tau$ irrational, and

$$
f(x):= \begin{cases}(x-\xi)^{-\gamma}, & x>\xi, 0<\gamma<1, \\ 0, & x \leq \xi,\end{cases}
$$

then for almost all $\tau, G_{n} f \rightarrow I(w f)$ if $\gamma<\frac{1}{2}$ and for all $\tau, G_{n} f \nrightarrow I(w f)$ if $\gamma \geq \frac{1}{2}[8,15]$. Analogous results hold for Radau and Lobatto rules with respect to $w$ [7].

A similar situation occurs in piecewise polynomial PIR's in that we ignore the singularity when $\xi=-1$ and avoid it when $\xi>-1[10]$.

\section{Convergence Results. I}

We first remark that if $f \in M_{d}(\xi ; k)$ and $k f \in D T\left(N_{\delta}(\lambda)\right)$, then $I(k f ; \lambda)$ exists, since we can write

$$
I(k f ; \lambda)=\int_{-1}^{M} k(x) \frac{f(x)}{x-\lambda} d x+f_{M}^{1} k(x) \frac{f(x)}{x-\lambda} d x,
$$

where $M:=(\xi+\lambda) / 2$. The first integral exists since $|f(x) /(x-\lambda)| \leq$ $(\lambda-M)^{-1}|f(x)|$, while the second integral exists as a CPV integral. In general we shall assume that both $k$ and $f$ separately are in $D T\left(N_{\delta}(\lambda)\right)$.

We shall now investigate the convergence of the interpolatory integration rules for CPV integrals given by (9) and (7) for singular functions $f \in M_{d}(\xi ; k)$. The first thing we must assume about these rules is that they converge for functions belonging to some smoothness class. Thus, if $k=w \in$ GSJ and $x_{i n}$ are the zeros of (3), then, by (13), it suffices that $g \in L D(1) \cap D T\left(N_{\delta}(\lambda)\right)$ for $I_{n}(w g ; \lambda)$ to converge to $I(w g ; \lambda)$, where

$$
L D(\nu)=\left\{f: \omega_{J}(f ; \delta) \log ^{\nu} \delta^{-1}=o(1), \delta \rightarrow 0^{+}, \nu>0\right\},
$$

and similarly, for the same points $x_{i n}$, if an arbitrary $k \in D T\left(N_{\delta}(\lambda)\right)$ satisfies (14), for $I_{n}(k g ; \lambda)$ to converge to $I(k g ; \lambda)$. For the more general case, using 
(11), we require at least that $f \in L D(2)$ to insure convergence, since on the one hand, $L D(1) \supset D T(J) \supset L D(1+\eta), \eta>0$, and on the other hand, the slowest rate of growth of $\Lambda\left(X_{n}\right)$ is of the order of $\log n$. This rate is achieved, for example, when $x_{i n}$ are the zeros of $T_{n}(x)$ or of $\left(1-x^{2}\right) U_{n-2}(x)$, the ClenshawCurtis points. For other sets of points, $X_{n}$, we may need more smoothness in $g$. Thus, if $x_{i n}$ are the zeros of the Legendre polynomials $P_{n}^{(0,0)}(x)$, then $\Lambda\left(X_{n}\right) \sim n^{1 / 2}$, and we require that $g \in H_{1 / 2+\eta}$ for some $\eta>0$, where

$$
H_{\mu}:=\left\{f: \omega_{J}(f ; \delta) \leq B \delta^{\mu}, B>0\right\}
$$

In any event, for a given $k$ and $X_{n}$, we require a certain amount of smoothness in $g$ to ensure convergence. This smoothness is a global smoothness on $J$ in contrast to the local smoothness required to ensure the existence of $I(k g ; \lambda)$. If $f \in M_{d}(\xi ; k)$, we cannot have this global smoothness but we shall instead assume that $f$ has the required smoothness in every closed subinterval $\Delta \subset$ $(\xi, 1]$. We can now state our convergence theorem for $I_{n}(k f ; \lambda)$, which we follow by several corollaries.

Theorem 1. Let $f \in M_{d}(\xi ; k)$ and assume that $k$ and the sets $X_{n}$ are such that $I_{n}(k g ; \lambda) \rightarrow I(k g ; \lambda)$ for all $g$ belonging to some smoothness class $S(J) \subset$ $C(J)$. Assume that $f$ belongs to $S(\Delta)$ for all closed intervals $\Delta \subset(\xi, 1]$ and that, given any $\varepsilon>0$ and any $\eta \in(\xi, 1]$ we can find $a \beta \in(\xi, \eta)$ such that

$$
\sum_{\xi<x_{i n} \leq \beta}\left|w_{i n}(k ; \lambda)\right| F\left(x_{i n}\right)<\varepsilon
$$

for all $n$ sufficiently large, where $F$ is the function given in (4). Then

$$
I_{n}(k f ; \lambda) \rightarrow I(k f ; \lambda) \quad \text { as } n \rightarrow \infty .
$$

Proof (cf. [10, p. 152]). Let $\varepsilon>0$ be given. Since $k F \in L_{1}(J)$, there exists $\eta \in(\xi, M]$ such that $\int_{\xi}^{\eta}|k(x) F(x)| d x<\varepsilon$, where $M:=(\xi+\lambda) / 2$. Choose $\beta \in(\xi, \eta)$ such that (27) holds. We then have $\int_{\xi}^{\beta}|k(x) F(x)| d x<\varepsilon$. Define now a function $\bar{f}_{\beta} \in S(J)$ by

$$
\bar{f}_{\beta}(x):= \begin{cases}f(x), & \beta \leq x \leq 1, \\ h(x), & \xi \leq x \leq \beta \\ 0, & -1 \leq x \leq \xi\end{cases}
$$

where $h(x)$ is a function of smoothness class $S[\xi, \beta]$ such that $\bar{f}_{\beta} \in S(J)$ and $|h(x)| \leq B F(x)$ in $[\xi, \beta]$ for some $B>0$. For example, if $S(J)=H_{\mu}$, we can choose $h$ to be a linear function such that $h(\xi)=0$ and $h(\beta)=f(\beta)$, in which case $B=1$. 
We now have

$$
\begin{aligned}
& \left|I_{n}(k f ; \lambda)-I(k f ; \lambda)\right| \\
& \quad \leq\left|I_{n}\left(k\left(f-\bar{f}_{\beta}\right) ; \lambda\right)\right|+\left|I_{n}\left(k \bar{f}_{\beta} ; \lambda\right)-I\left(k \bar{f}_{\beta} ; \lambda\right)\right|+\left|I\left(k\left(f-\bar{f}_{\beta}\right) ; \lambda\right)\right| \\
& \quad \leq \sum_{\xi<x_{i n} \leq \beta}\left|w_{i n}(k ; \lambda)\right|\left(\left|f\left(x_{i n}\right)\right|+\left|\bar{f}_{\beta}\left(x_{i n}\right)\right|\right)+\left|I_{n}\left(k \bar{f}_{\beta} ; \lambda\right)-I\left(k \bar{f}_{\beta} ; \lambda\right)\right| \\
& \quad+\left|\int_{\xi}^{\beta} \frac{k(x) f(x)}{x-\lambda} d x\right|+\left|\int_{\xi}^{\beta} \frac{k(x) \bar{f}_{\beta}(x)}{x-\lambda} d x\right| \\
& \quad \leq(1+B) \varepsilon+\left|I_{n}\left(k \bar{f}_{\beta} ; \lambda\right)-I\left(k \bar{f}_{\beta} ; \lambda\right)\right|+(M-\lambda)^{-1}(1+B) \varepsilon .
\end{aligned}
$$

Since $\bar{f}_{\beta} \in S(J), I_{n}\left(k \bar{f}_{\beta} ; \lambda\right) \rightarrow I\left(k \bar{f}_{\beta} ; \lambda\right)$ as $n \rightarrow \infty$, which proves $(28)$.

Corollary 1.1. Let there be given a sequence of sets $X_{n}, k \in D T\left(N_{\delta}(\lambda)\right) \cap L_{1}(J)$ and $f \in M_{d}(\xi ; k)$ such that the companion rules $\left|I_{n}\right|(k F) \rightarrow I(|k| F)$, where $F$ is given in (4). Assume that $I_{n}(k g ; \lambda) \rightarrow I(k g ; \lambda)$ for all $g \in S(J)$, that $f \in S(\Delta)$ for all closed intervals $\Delta \subset(\xi, 1]$ and that

$$
I\left(k P_{n} ; \lambda\right) / P_{n}^{\prime}\left(x_{i n}\right)=O\left(w_{i n}(k)\right)
$$

for all indices $i$ such that $\xi<x_{i n}<\xi+\delta$ for some $\delta>0$, where $P_{n}$ is given by (8). Then (28) holds.

Proof. By Theorem C, $\sum_{\xi<x_{i n} \leq \beta}\left|w_{i n}(k)\right| F\left(x_{i n}\right)<\varepsilon$, where we can choose $\beta \leq$ $M$. By (29),

$$
\left|w_{\text {in }}(k ; \lambda)\right| \leq C\left|w_{\text {in }}(k)\right| /\left|x_{\text {in }}-\lambda\right|<C(M-\lambda)^{-1}\left|w_{\text {in }}(k)\right| .
$$

Hence, by Theorem 1, (28) holds.

Corollary 1.2. Let $w \in G S J$ and let $x_{i n}$ be the zeros of (3). Assume that $f \in M_{d}(\xi ; w) \cap D T\left(N_{\delta}(\lambda)\right)$ and that $f \in L D(1)$ in all closed subintervals $\Delta \subset(\xi, 1]$. Let $\xi=\cos \pi \tau$.

(a) If $\xi>-1$, then $I_{n}^{\prime}(w f ; \lambda) \rightarrow I(w f ; \lambda)$ as $n \rightarrow \infty$.

(b) If $\xi=-1$ and $\gamma_{0} \leq \frac{1}{2}$ in (2), then (28) holds with $k=w$.

(c) If $w$ is the Jacobi weight function (26) and $\tau<1$ is a positive rational number, then (28) holds. If $\tau$ is irrational and $|f(x)| \leq B(x-\xi)^{-\mu}$ with $\mu<\frac{1}{2}$, then (28) holds for almost all $\xi$. Here also, $k=w$ in (28).

Proof. Since $I_{n}(w f)$ is a Gauss, Radau or Lobatto rule, we have by the remarks following Corollary C.2 that $I_{n}^{\prime}(w F) \rightarrow I(w F)$ in case (a) and $I_{n}(w F) \rightarrow$ $I(w F)$ in cases (b) and (c), where $F$ is the function specified in (4). Furthermore, the weights $w_{\text {in }}(w)$ are the Gauss, Radau or Lobatto weights $\mu_{\text {in }}$ which are positive. Hence, it remains to show that $I_{n}^{\prime}(w g ; \lambda) \rightarrow I(w g ; \lambda)$ for all $g \in L D(1) \cap D T\left(N_{\delta}(\lambda)\right)$ in case (a) and that (29) holds in all three cases. To this end, we quote some results about the quantities appearing in (29) for the Gauss case. As in [7,9], similar results hold for the Radau and Lobatto cases. 
From [5, (19)] we have that

$$
w_{\text {in }}(w)=\mu_{i n} \sim n^{-1} w\left(x_{i n}\right)\left(1-x_{i n}^{2}\right)^{1 / 2}
$$

and from $[5,(17)]$ that

$$
1+x_{i n} \sim n^{-2}, \quad i=1,2, \ldots, l .
$$

From [4, p. 170] we have that

$$
1 / p_{n}^{\prime}\left(x_{i n}\right)=A_{n-1} A_{n}^{-1} \mu_{i n} p_{n-1}\left(x_{i n}\right), \quad A_{n-1} A_{n}^{-1}<1 .
$$

From [5, (22)],

$$
\left|p_{n-1}\left(x_{i n}\right)\right| \sim w\left(x_{i n}\right)^{-1 / 2}\left(1-x_{i n}^{2}\right)^{1 / 4},
$$

and from [2], we have that $q_{n}(\lambda):=I\left(p_{n} ; \lambda\right)=O(1)$. From these it follows that (29) holds, since

$$
\begin{aligned}
\left|I\left(w P_{n} ; \lambda\right) / P_{n}^{\prime}\left(x_{i n}\right)\right| & =\left|q_{n}(\lambda) / p_{n}^{\prime}\left(x_{i n}\right)\right| \\
& =O\left(\mu_{i n} w\left(x_{i n}\right)^{-1 / 2}\left(1-x_{\text {in }}^{2}\right)^{1 / 4}\right) \\
& =O\left(\mu_{\text {in }}\right) \quad \text { if }\left\{\begin{array}{l}
\xi>-1, \\
\xi=-1 \text { and } \gamma_{0} \leq 1 / 2 .
\end{array}\right.
\end{aligned}
$$

Furthermore, since $\mu_{\kappa n}=o(1)$, it follows that $w_{\kappa n}(w ; \lambda)=o(1)$, so that $I_{n}^{\prime}(w g ; \lambda) \rightarrow I(w g ; \lambda)$, which completes the proof.

We see that the case $\xi=-1$, which is a favorable case as far as convergence of $I_{n}(w f)$ is concerned, is not favorable for the convergence of $I_{n}(w f ; \lambda)$ or for that matter, of $I_{n}^{\prime}(w f ; \lambda)$. This is so since $q_{n}(\lambda) / p_{n}^{\prime}\left(x_{i n}\right)$ does not tend to zero as quickly as $\mu_{\text {in }}$ in the neighborhood of -1 . Even though we have only that $q_{n}(\lambda)=O(1)$, we have in fact that $q_{n}(\lambda)$ oscillates between two finite bounds, so that $q_{n}(\lambda) \neq o(1)$. Thus, for the normalized Legendre case, we have $[14,(8.21 .19)]$

$$
q_{n}(\cos \theta)=(\pi(2 n+1) / 4 n \sin \theta)^{1 / 2} \cos \{(n+1 / 2) \theta+\pi / 4\}+O\left(n^{-1}\right),
$$

and similar expressions exist for the Jacobi case.

\section{Convergence results. II}

We now investigate the convergence of $\widehat{I}_{n+1}(k f ; \lambda)$ to $I(k f ; \lambda)$, or equivalently, that of $I_{n}\left(k f_{\lambda}\right)$ to $I\left(k f_{\lambda}\right)$. As indicated above, we need not restrict our attention to IPIR's $I_{n}(k g)$ but can consider arbitrary PIR's $\bar{I}_{n}(k g)$, in particular the generalized piecewise polynomial PIR's studied in [10]. Thus, we state our main theorem for arbitrary PIR's.

Theorem 2. Assume that $\bar{I}_{n}\left(k g_{\lambda}\right) \rightarrow I\left(k g_{\lambda}\right)$ for all $g_{\lambda} \in P C[J]$ and that $\left|\bar{I}_{n}\right|(k F) \rightarrow I(|k| F)$ if $f \in M_{d}(\xi ; k)$, where $F$ is defined in (4). Then $\bar{I}_{n}\left(k f_{\lambda}\right)$ $\rightarrow I\left(k f_{\lambda}\right)$ 
Proof. If $\left|\bar{I}_{n}\right|(k F) \rightarrow I(|k| F)$, then by Theorem C, $\sum_{\xi<x_{i n} \leq \beta}\left|\bar{w}_{i n}(k)\right| F\left(x_{i n}\right)<$ $\varepsilon$. Hence,

$$
\sum_{\xi<x_{i n} \leq \beta}\left|\bar{w}_{i n}(k)\right|\left|f\left(x_{i n}\right)-f(\lambda)\right| /\left|x_{i n}-\lambda\right|<B \varepsilon,
$$

so that by Theorem $\mathrm{C}, \bar{I}_{n}\left(k f_{\lambda_{\lambda}}\right) \rightarrow I\left(k f_{\lambda_{\lambda}}\right)$.

We observe that $f_{\lambda}$ has possible singularities at $\xi$ and $\lambda$. If these are such that a sequence of PIR's which ignore the singularities converges, then we can use such rules, but it may turn out that we will have convergence only when we avoid the singularities. If $\xi=-1$, we can generally ignore the singularity at $\xi$. If $\xi \neq-1$, we may be able to ignore the singularity in some special cases but generally we must avoid the singularity. As for the point $\lambda$, since it is always an interior point, we must in general avoid the singularity, although in special situations, we can ignore it [8].

Before we apply Theorem 2 to derive some results on the convergence of $\widehat{I}_{n+1}(k f ; \lambda)$ to $I(k f ; \lambda)$ when $f \in M_{d}(\xi ; k)$, we observe that we can use Theorems 2 and 3 and Corollaries 3 and 4 in [10] to establish conditions for the convergence of $\bar{I}_{n}\left(k f_{\lambda}\right)$ to $I\left(k f_{\lambda}\right)$ when the $\bar{I}_{n}\left(k f_{\lambda}\right)$ are generalized piecewise polynomial PIR's. Here also, we ignore the singularity when $\xi=-1$ and avoid it when $\xi \neq-1$ and for all $\lambda$. We will not enter into the details here.

We now state our first corollary.

Corollary 2.1. Let $w \in A$, let $x_{\text {in }}$ be the roots of (3) and let $I_{n}(k h)$ be an IPIR that converges for all $h \in R[J]$. If $f \in M_{d}(\xi ; k) \cap H_{1}\left(N_{\delta}(\lambda)\right)$ and $\left|I_{n}\right|(k F) \rightarrow$ $I(|k| F)$, where $F$ is defined in (4), then $I_{n}\left(k f_{\lambda}\right) \rightarrow I\left(k f_{\lambda}\right)$.

Proof. $I_{n}(k h) \rightarrow I(k h)$ for all $h \in R[J]$ implies that $I_{n}\left(k g_{\lambda}\right) \rightarrow I\left(k g_{\lambda}\right)$ if $g \in H_{1}\left(N_{\delta}(\lambda)\right) \cap R[J]$.

We now introduce some notation similar to $I_{n}^{\prime}$. Let $x_{\sigma n}$ be the point in $X_{n}$ closest to $\lambda$, where, if two points are closest to $\lambda$, we choose $x_{\sigma n}>\lambda$. Then $I_{n}^{*}(w g):=I_{n}(w g)-\bar{w}_{\sigma n} g\left(x_{\sigma n}\right)$ and $I_{n}^{\prime *}(w g):=I_{n}^{\prime}(w g)-\bar{w}_{\sigma n} g\left(x_{\sigma n}\right)=$ $I_{n}^{*}(w g)-w_{\kappa n} g\left(x_{\kappa n}\right)$.

Corollary 2.2. Let $w \in A$ and let $x_{\text {in }}$ be the roots of (3). Let $k=w$ and assume $0<b \leq w(x) \leq B$ for $x \in N_{\delta}(\lambda) \cup N_{\delta}(\xi)$. If $f \in M_{d}(\xi ; w)$ and $\xi>-1$, then $I_{n}^{\prime *}\left(w f_{\lambda}\right) \rightarrow I\left(w f_{\lambda}\right)$. If $f \in H_{\mu}\left(N_{\delta}(\lambda)\right), \mu>\frac{1}{2}$, and $|f(x)|<B_{1}(x-\xi)^{-\nu}$, $\nu<\frac{1}{2}$, for $\xi<x<\xi+\delta$, then for almost all pairs $(\xi, \lambda), I_{n}\left(w f_{\lambda}\right) \rightarrow I\left(w f_{\lambda}\right)$.

Proof. In this case, $w_{i n}(w)=\mu_{i n}$. Since $0<b \leq|w(x)| \leq B$ in $N_{\delta}(\lambda) \cup$ $N_{\delta}(\xi), x_{j+1, n}-x_{j n} \sim 1 / n$ and $\mu_{j n} \sim 1 / n$ for all $x_{j n}$ in $N_{\delta}(\lambda) \cup N_{\delta}(\xi)$ [7]. Since $I_{n}(w h)$ is a Gauss, Radau or Lobatto rule, $I_{n}^{\prime}(w F)=\left|I_{n}^{\prime}\right|(w F) \rightarrow$ $I(|w| F)=I(w F)$. Furthermore, $I_{n}^{*}\left(w g_{\lambda}\right) \rightarrow I\left(w g_{\lambda}\right)$ for every $g_{\lambda} \in R[J]$. Hence $I_{n}^{\prime *}\left(w f_{\lambda_{i}}\right) \rightarrow I\left(w f_{\dot{\lambda}}\right)$. If the additional conditions in the corollary hold, then using the results in [7], we see that we can drop the prime and the asterisk from $I^{\prime *}$ for almost all pairs $(\xi, \lambda)$. 
Corollary 2.3. Let $w \in G S J$ and $x_{\text {in }}$ be the zeros of (3). If $f \in M_{d}(\xi ; w)$ and $\xi>-1$, then $\widehat{I}_{n+1}^{*}(w f ; \lambda) \rightarrow I(w f ; \lambda)$ and if $\xi=-1$, then $\widehat{I}_{n+1}^{*}(w f ; \lambda) \rightarrow$ $I(w f ; \lambda)$.

Proof. If $\xi>-1$ and $\xi, \lambda \neq t_{j}, j=1, \ldots, p$, as we have always assumed, then there exists $\delta>0$ such that $0<b \leq w(x) \leq B$ for $x \in N_{\delta}(\lambda) \cap N_{\delta}(\xi)$. If $\xi=-1$, we know that $I_{n}^{*}\left(w f_{\lambda}\right) \rightarrow I\left(w f_{\lambda}\right)$ by taking $\bar{I}_{n}\left(w f_{\lambda}\right)$ to be $I_{n}^{*}\left(w f_{\lambda}\right)$.

Corollary 2.4. Let $w$ be the Jacobi weight function (26), let $x_{i n}$ be the zeros of (3) and let $k \in D T\left(N_{\delta}(\lambda)\right)$ satisfy (14). Assume that $f \in M_{d}(\xi ; k)$ and that $k$ is continuous and of bounded variation in $N_{\delta}(\xi) \cup N_{\delta}(\lambda)$. Then $\widehat{I}_{n+1}^{*}(k f ; \lambda) \rightarrow$ I $(k f ; \lambda)$. If $\xi(\lambda)=\cos \pi p / q$, we can drop the prime (asterisk). If $f \in H_{\mu}$ in $N_{\delta}(\lambda), \mu>\frac{1}{2}$, we can drop the asterisk for almost all $\lambda$. If $|f(x)| \leq B(x-\xi)^{-\nu}$, $\nu<\frac{1}{2}$, for $\xi<x<\xi+\delta$, we can drop the prime for almost all $\xi$.

Proof. By Theorem 2 in [13], if $x$ is a fixed point in $(-1,1)$ and $j$ is the unique integer such that $x_{j n} \leq x<x_{j+1, n}$ and $k$ satisfies (14) and is continuous and of bounded variation in an open interval $X$ which contains $x$, then $n w_{i n}(k) \rightarrow \pi k(x)\left(1-x^{2}\right)^{1 / 2}$ as $n \rightarrow \infty$, and the convergence is uniform in any closed interval in $X$ containing $x$. This implies that $w_{i n}(k) \sim \mu_{\text {in }}$, so that we can apply the same reasoning as used to prove Corollary 2.3 to show that $\widehat{I}_{n+1}^{*}(k f ; \lambda) \rightarrow I(k f ; \lambda)$. The other results follow from convergence results for Gauss, Radau or Lobatto rules based on zeros of Jacobi polynomials in which we can ignore the singularity instead of avoiding it in the circumstances mentioned in the corollary.

\section{BIBLIOGRAPHY}

1. G. Criscuolo and G. Mastroianni, On the convergence of an interpolatory product rule for evaluating Cauchy principal value integrals, Math. Comp. 48 (1987), 725-735.

2. G. Criscuolo, G. Mastroianni, and P. Nevai, Associated generalized Jacobi functions and polynomials (submitted).

3. G. Monegato, The numerical evaluation of one-dimensional Cauchy principal value integrals, Computing 29 (1982), 337-354.

4. P. Nevai, Orthogonal polynomials, Mem. Amer. Math. Soc. No. 213 (1979).

5. P. Nevai and P. Vértesi, Mean convergence of Hermite-Fejér interpolation, J. Math. Anal. Appl. 105 (1985), 26-58.

6. P. Rabinowitz, Some practical aspects in the numerical evaluation of Cauchy principal value integrals, Internat. J. Comput. Math. 20 (1986), 283-298.

7. __ Rates of convergence of Gauss, Lobatto, and Radau integration rules for singular integrands, Math. Comp. 47 (1986), 625-638.

8. __ Numerical integration in the presence of an interior singularity, J. Comput. Appl. Math. 17 (1987), 31-41.

9. $\ldots$, On an interpolatory product rule for evaluating Cauchy principal value integrals, BIT 29 (1989), 347-355.

10. P. Rabinowitz and I. H. Sloan, Product integration in the presence of a singularity. SIAM J. Numer. Anal. 21 (1984), 149-166. 
11. P. Rabinowitz and W. E. Smith, Interpolatory product integration for Riemann-integrable functions, J. Austral. Math. Soc. Ser. B 29 (1987), 195-202.

12. I. H. Sloan and W. E. Smith, Properties of interpolatory product integration rules, SIAM J. Numer. Anal. 19 (1982), 427-442.

13. W. E. Smith and I. H. Sloan, Product-integration rules based on the zeros of Jacobi polynomials, SIAM J. Numer. Anal. 17 (1980), 1-13.

14. G. Szegö, Orthogonal polynomials, Amer. Math. Soc. Colloq. Publ., vol. 23, Amer. Math. Soc., Providence, R. I., 1959.

15. P. Vértesi, Remarks on convergence of Gaussian quadrature for singular integrals, Acta Math. Hungar. 53 (1989), 399-405.

Department of Applied Mathematics and Computer Science, The Weizmann Institute of SCIENCE, REHOVot 76100, ISRAEl.E-mail: maweintr@weizmann.bitnet 